\title{
Regenerative effect of platelet-rich fibrin on articular cartilage defects in an experimental rat model
}

\author{
Hasan Metineren๑, Turan Cihan Dülgeroğlu๑ \\ Department of Orthopaedics and Traumatology, Dumlupınar University School of Medicine, Kütahya, Turkey
}

DOI: $10.18621 /$ eurj.373385

\begin{abstract}
Objectives: Different materials are currently being used experimentally to accelerate cartilage healing and to obtain hyaline cartilage. We aimed to investigate the effect of platelet-rich fibrin on joint cartilage healing.

Methods: Twenty rats were used in this study. platelet-rich fibrin was obtained from 4 rats, and the remaining 16 rats were randomly divided into two groups ( 4 and 8 weeks). Both knee joints were prepared, and an osteochondral defect was created at the femoral condyle in all rats. platelet-rich fibrin was placed into the osteochondral defect of the right knee, while the left knee joint was used as a control. Groups were sacrificed after 4 and 8 weeks, respectively.

Results: According to the O'Driscoll score, there were no significant differences between the right and left knee scores in group 4 weeks $(p=0.820)$. However, the right knee scores were significantly different than the left knee scores in group 8 weeks $(p<0.001)$. Defects were completely filled with cartilage tissue after 8 weeks in the platelet-rich fibrin group.

Conclusions: Similar to its role in various tissues, platelet-rich fibrin is an effective biomaterial that enhances the healing of osteochondral defects.
\end{abstract}

Keywords: Articular cartilage, chondral healing, experimental rat model, hyaline cartilage, platelet-rich fibrin

Received: January 1, 2018; Accepted: July 1, 2018; Published Online: July 12, 2018

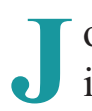
oint cartilage is a unique tissue, that is involved in joint motion and resistance during weight bearing. However, the lack of primary vascular nutrition renders joint cartilage repair after damage difficult [1]. Chondrocytes have a limited potential for migration to damaged sites, and untreated cartilage lesions can result in degenerative joint diseases. The limited treatment options for focal cartilage defects include microfracture procedures, use of biomaterials, and scaffolds. During microfracture, perforation of the subchondral bone plate is performed to stimulate bone marrow, and to enhance the migration of non-differ- entiated bone marrow-derived multipotent stem cells to the defective site [2]. The use of biomaterials is widespread, but there is no consensus as to its effectiveness. The cellular and acellular biomaterials that are used currently both have advantages. Acellular biomaterials such as collagen, hyaluronic acid, synthetic polymers, alginate, and chitosan are relatively cheaper than cellular cultures, and are not associated with donor site issues. However, an ideal biomaterial should be rich in cells and growth factors. Platelet-rich fibrin (PRF), a second-generation platelet-rich biomaterial [3] that is rich in platelets, neutrophils, and cir-

Address for correspondence: Hasan Metineren, Assistant Professor, Dumlupinar University School of Medicine, Department of Orthopaedics and Traumatology, Evliya Çelebi Campus, 43100 Kütahya, Turkey E-mail:drhmetin19@gmail.com,Tel: +902742316660/2109 
culating stem cells, was developed by Choukron [46]. PRF contains several growth factors and cytokines, including transforming growth factor (TGF)- $\beta$, vascular endothelial growth factor (VEGF), insulin-like growth factor (IGF)-I and IGF-II, platelet-derived growth factor (PDGF), the inflammatory cytokines interleukin (IL)-1, IL-6, tumour necrosis factor (TNF)$\alpha$, and the anti-inflammatory cytokines IL- 4 and IL-10 $[7,8]$. PRF is the highly effective biomaterial in terms of cellular content, growth factors, and inflammatory cytokines compared with platelet-rich plasma (PRP), plasma rich in growth factors (PRGF), advanced platelet-rich fibrin (A-PRF), and concentrated growth factors (CGF) [9]. Several authors have shown that growth factors, including PDGF and TGF- $\beta$, which are constantly released from PRF for at least one week and up to 28 days, support its accelerating effect on tissue healing $[4-7,10]$. PRF is obtained by centrifugation of fresh whole blood; therefore, it does not cause allergic reactions. In this experimental study, we investigated the histological effects of PRF on cartilage regeneration.

\section{METHODS}

This study was approved by the University Ethical Committee of Laboratory Animals Care and Use $(04 / 01 / 2014-09 / 01 / 2014)$. The study included twenty mature male Sprague-Dawley rats (average length 18 $\mathrm{cm}$ ) weighing approximately 300 grams. To obtain PRF, blood samples were collected from four rats using conventional glass tubes without anticoagulants.

\section{Preparation of PRF}

The collected blood was centrifuged at $2700 \mathrm{rpm}$ for 12 minutes. Then, the PRF matrix was immediately withdrawn from the tube, and the red blood cell (RBC) fraction was eliminated. A large number of platelets were trapped between the yellow fibrin section and the RBC section with the buffy coat section [7] (Figure 1). When PRP was obtained, first, donors blood was treated with anticoagulants. Then the primary centrifugation was applied (at $1500 \mathrm{rpm}$ for ten minutes) to separate the red cells and the plasma. Second centrifugation was carried out for the concentration of the platelets. $(3000 \mathrm{rpm}$ for ten minutes). It has been reported in the literature by the authors who define the original method of PRF preparation that no allogenic reaction occurs $[7,8]$. There is no allergic or histological abnormality was observed due to use of allogeneic PRF.

\section{Surgical Procedure}

The animals were housed in the Laboratory Animal Care-Augmentation facility of our university in a temperature-controlled room $\left(20-22^{\circ} \mathrm{C}\right)$, on a 12 hour light/dark cycle. They were provided with rat pellets and water ad libitum. Four animals were housed together per standard cage. The 16 rats used in this study were randomly divided into two groups (groups 4 and 8 weeks). Anaesthesia was induced via an intraperitoneal injection of ketamine $(50 \mathrm{mg} / \mathrm{kg})$ and xylazine hydrochloride $(10 \mathrm{mg} / \mathrm{kg})$. Both knees of each animal were prepared for aseptic surgery with chlorhexidine gluconate. An anterior longitudinal incision was made in the knee, and the patella was

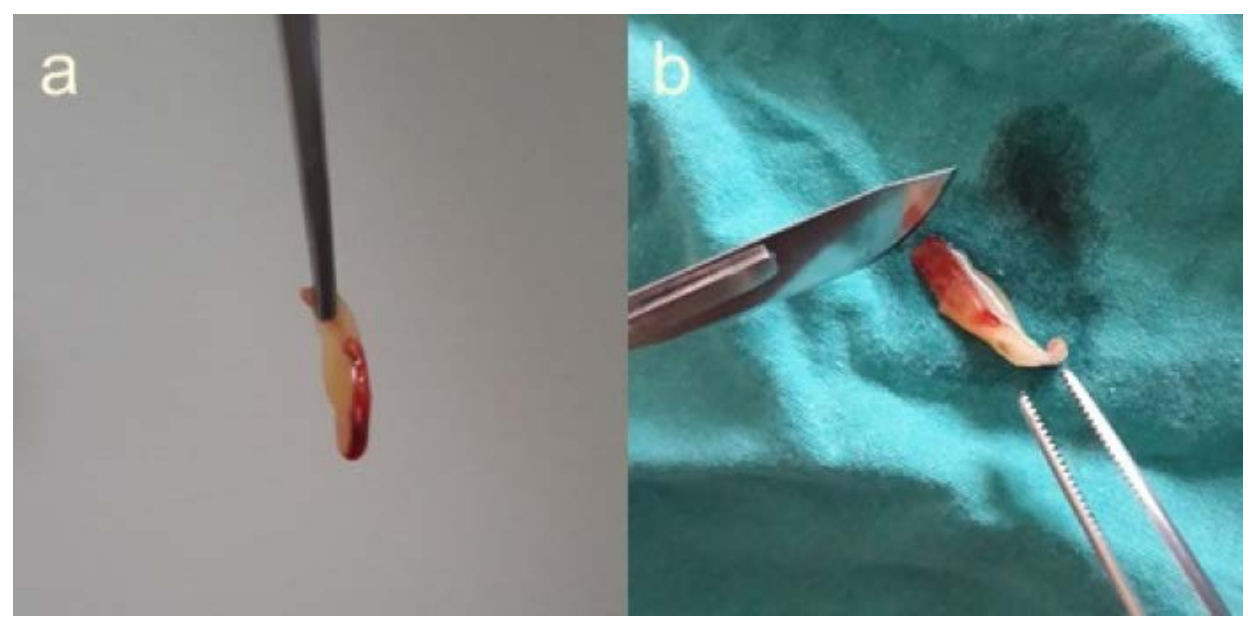

Figure 1. The obtained PRF was separated into eight equal parts $(a, b)$. 
Table 1. The O'Driscoll histological cartilage repair scale

\begin{tabular}{|c|c|}
\hline Characteristics & Score \\
\hline \multicolumn{2}{|l|}{ Nature of predominant tissue } \\
\hline \multicolumn{2}{|l|}{ Cellular morphology } \\
\hline Hyaline articular cartilage & 4 \\
\hline Incompletely differentiated mesenchyme & 2 \\
\hline Fibrous tissue or bone & 0 \\
\hline \multicolumn{2}{|l|}{ Staining of the matrix } \\
\hline Normal or nearly normal & 3 \\
\hline Moderate & 2 \\
\hline Slight & 1 \\
\hline None & 0 \\
\hline \multicolumn{2}{|l|}{ Structural characteristics } \\
\hline \multicolumn{2}{|l|}{ Surface regularity } \\
\hline Smooth and intact & 3 \\
\hline Superficial horizontal lamination & 2 \\
\hline Fissures $25-100 \%$ of the thickness & 1 \\
\hline Severe disruption including fibrillation & 0 \\
\hline \multicolumn{2}{|l|}{ Structural integrity } \\
\hline Normal & 2 \\
\hline Slight disruption including cysts & 1 \\
\hline Severe disintegration & 0 \\
\hline \multicolumn{2}{|l|}{ Thickness } \\
\hline $100 \%$ of normal adjacent cartilage & 2 \\
\hline $50-100 \%$ of normal cartilage & 1 \\
\hline $0-50 \%$ of normal cartilage & 0 \\
\hline \multicolumn{2}{|l|}{ Bonding to the adjacent cartilage } \\
\hline Bonded at both ends of graft & 2 \\
\hline Bonded at one end or partially at both ends & 1 \\
\hline Not bonded & 0 \\
\hline \multicolumn{2}{|l|}{ Freedom from cellular changes of degeneration } \\
\hline \multicolumn{2}{|l|}{ Hypocellularity } \\
\hline Normal cellularity & 3 \\
\hline Slight hypocellularity & 2 \\
\hline Moderate hypocellularity & 1 \\
\hline Severe hypocellularity & 0 \\
\hline \multicolumn{2}{|l|}{ Chondrocyte clustering } \\
\hline No clusters & 2 \\
\hline$<25 \%$ of the cells & 1 \\
\hline $25-100 \%$ of the cells & 0 \\
\hline \multicolumn{2}{|l|}{$\begin{array}{l}\text { Freedom from degenerative changes in adjacent } \\
\text { cartilage }\end{array}$} \\
\hline $\begin{array}{l}\text { Normal cellularity, no clusters, and normal } \\
\text { staining }\end{array}$ & 3 \\
\hline $\begin{array}{l}\text { Normal cellularity, mild clusters, and slight } \\
\text { staining }\end{array}$ & 2 \\
\hline Mild or moderate hypocellularity, slight staining & 1 \\
\hline Severe hypocellularity, poor or no staining & 0 \\
\hline Total & 24 \\
\hline
\end{tabular}

dislocated laterally. An osteochondral defect was created at both femoral condyles using a $1.50 \mathrm{~mm}$ (0.057-inch)-diameter stainless Kirschner wire (Aysam Samsun, Turkey) to a depth of $2 \mathrm{~mm}$. This procedure was performed in all animals for both knees (Figure 2). The collected PRF was divided into eight equal portions and placed into the defective areas of the right knees, and the joint capsule was repaired.

Cartilage healing is a process that is completed in 6 weeks, similar to fracture healing. Evaluation was made at $4^{\text {th }}$ and $8^{\text {th }}$ weeks to investigate the early and late effects of PRF.Groups were sacrificed 4 and 8 weeks post-surgery, respectively, and the femoral condyles were harvested for histological evaluation.

\section{Histology and Immunohistochemistry}

The specimens were fixed in 10\% buffered neutral formalin and decalcified in acid solution for two weeks. Following decalcification, the specimens were dehydrated and subsequently embedded in paraffin wax. Sections $10 \mu \mathrm{m}$ thick were made through the centre of the defect. Cartilage sections were stained with hematoxylin and eosin stain for overall evaluation of the tissue. Histological depth measurement was not performed. Masson's trichrome stain was used for the evaluation of the osteochondral zone, and Toluidine blue stain was used to determine the presence of proteoglycan. The sections were blindly scored by two independent investigators (MHM and CK) and evaluated using the O'Driscoll scale [11] (Table 1).

\section{Statistical analysis}

The SPSS software version 22.0 Mining (IBM Corp., Armonk, NY, USA) was used for all analyses. Normally distributed data were analysed using the Shapiro-Wilk test, and variance homogeneity was assessed using the Levene test. Univariate analysis of variance (ANOVA) was used with bootstrap analysis to determine dependent quantitative variables in groups 4 and 8 weeks and for the left and right knees according to the O'Driscoll scores. Quantitative variables are expressed as means \pm standard deviations (SD), and categorical variables are expressed as $\mathrm{n}(\%)$. Variables were examined at a $95 \%$ confidence level, and $p<0.05$ was considered to indicate statistical significance.

\section{RESULTS}

The mean value of PRF in the right knees of 8 week group $(11.75 \pm 1.67)$ was significantly higher than that of 4 week group $(20.38 \pm 2.62)(p<0.001)$. 


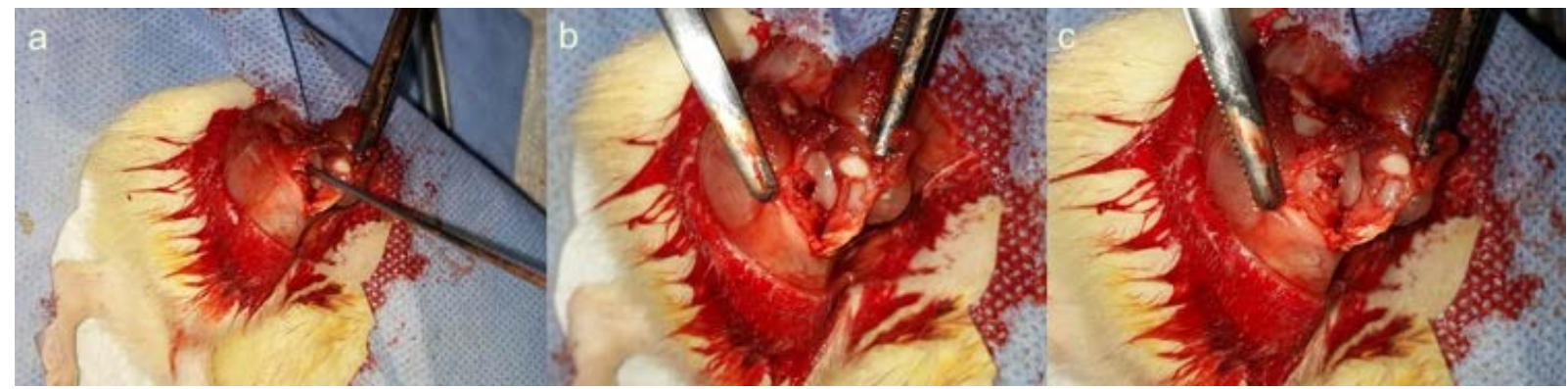

Figure 2. Osteochondral defects were created using a Kirschner wire at the femoral condyles and the right knee defects were subsequently filled with PRF (a-c).

Table 2. Comparison of the right and left knee scores in both groups

\begin{tabular}{lccc}
\hline Groups & Knee & $p_{\text {value }}^{1}$ \\
\hline & Mean \pm SD. / Max.-Min. & Mean \pm SD. / Max.-Min. & \\
\hline 4 weeks & $11.75 \pm 1.67 / 14-9$ & $12.00 \pm 2.27 / 15-8$ & 0.820 \\
8 weeks & $20.38 \pm 2.62 / 24-16$ & $12.25 \pm 2.05 / 16-10$ & $<\mathbf{0 . 0 0 1}$ \\
p value $^{2}$ & $<\mathbf{0 . 0 0 1}$ & 0.820 & \\
\hline
\end{tabular}

Univariate Analysis of Variance (ANOVA) (Bootstrap), SD =Standard deviation $p$ value $^{1}=4$ weeks versus 8 weeks groups, $p$ value ${ }^{2}=$ Right knee versus left knee

There were no significant differences between the control (left) knees of both groups $(p=0.820)$ (Table 2).

The O'Driscoll score of the PRF-treated group (right knees) $(20.38 \pm 2.62)$ was significantly higher than that of the control group $(12.25 \pm 2.05)$ during the eighth week $(p<0.001)$. There were no differences in the knees of group 4 weeks $(p=0.820)$ (Figure 3$)$.
Four weeks after surgery, the defects in both groups were filled with 50\% fibrocartilage. At the end of 8 weeks, the PRF placed in the defective zone of the right knee was completely resorbed, and the defect was filled with hyaline cartilage (Figure 4). In the control group, differentiated mesenchymal cells and fibrosis cartilage tissue were predominant in some sections (Figure 5).

\section{O'DRISCOLL SCORES}

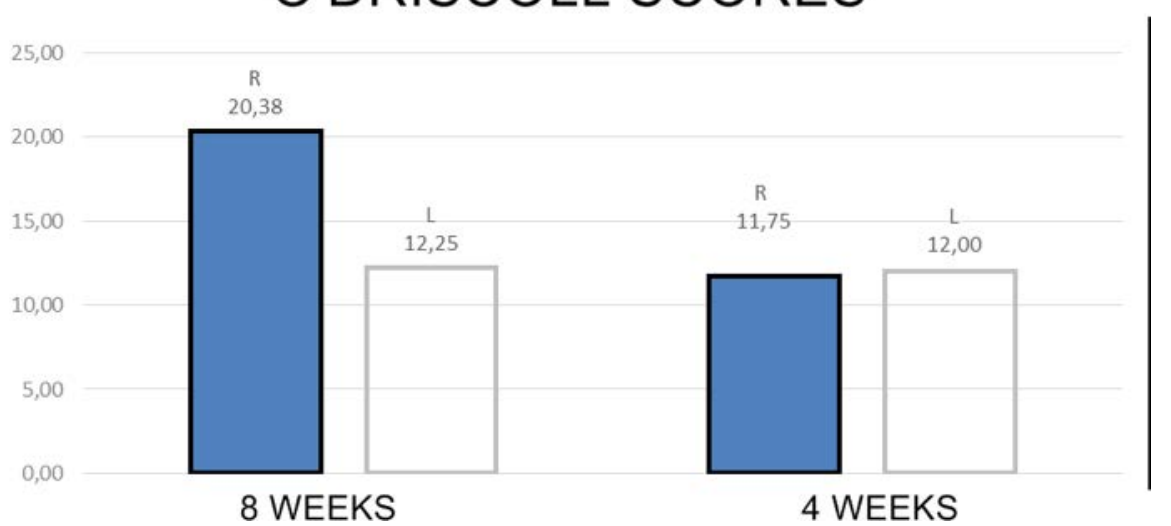

Figure 3. Distribution of right and left knee scores according to the O'Driscoll scoring system in groups A and B. 


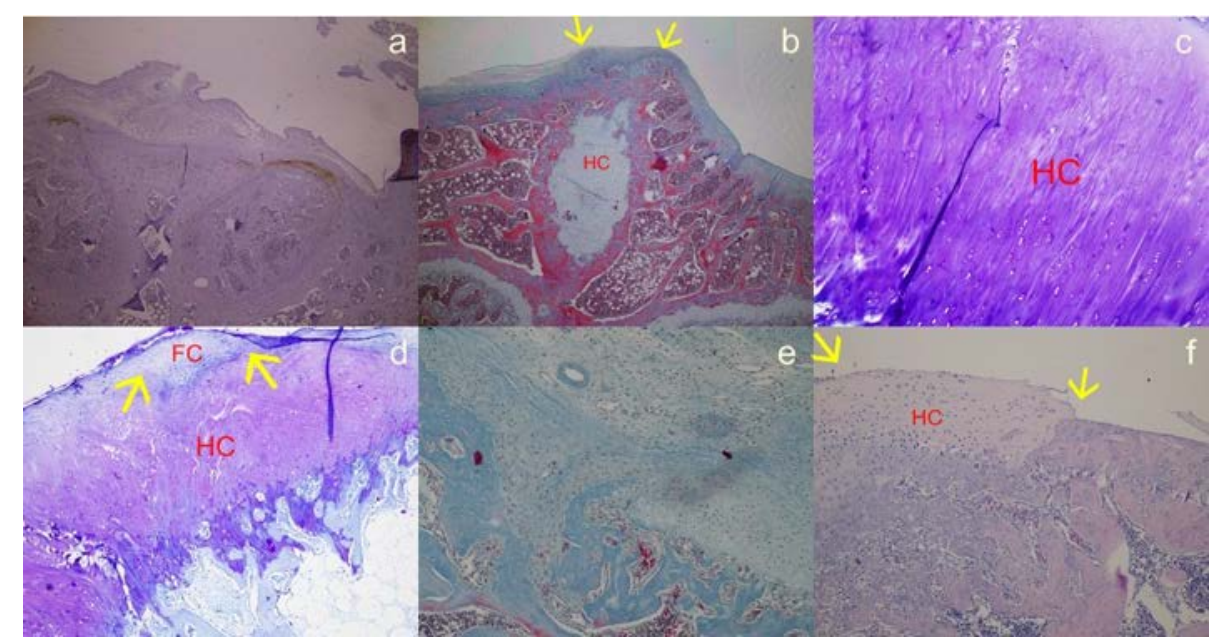

Figure 4. Histological evaluations in the PRF-treated knees of group B (a-f). (a) Hyaline cartilage healing; collagen II $(\times 10)$, (b, e) Masson trichrome $(\times 20, \times 40)$, (c, d) a fully healed defect; Toluidine blue $(\times 40, \times 20)$, and (f) smooth surface shown with hematoxylin \& eosin $(\times 20)$.

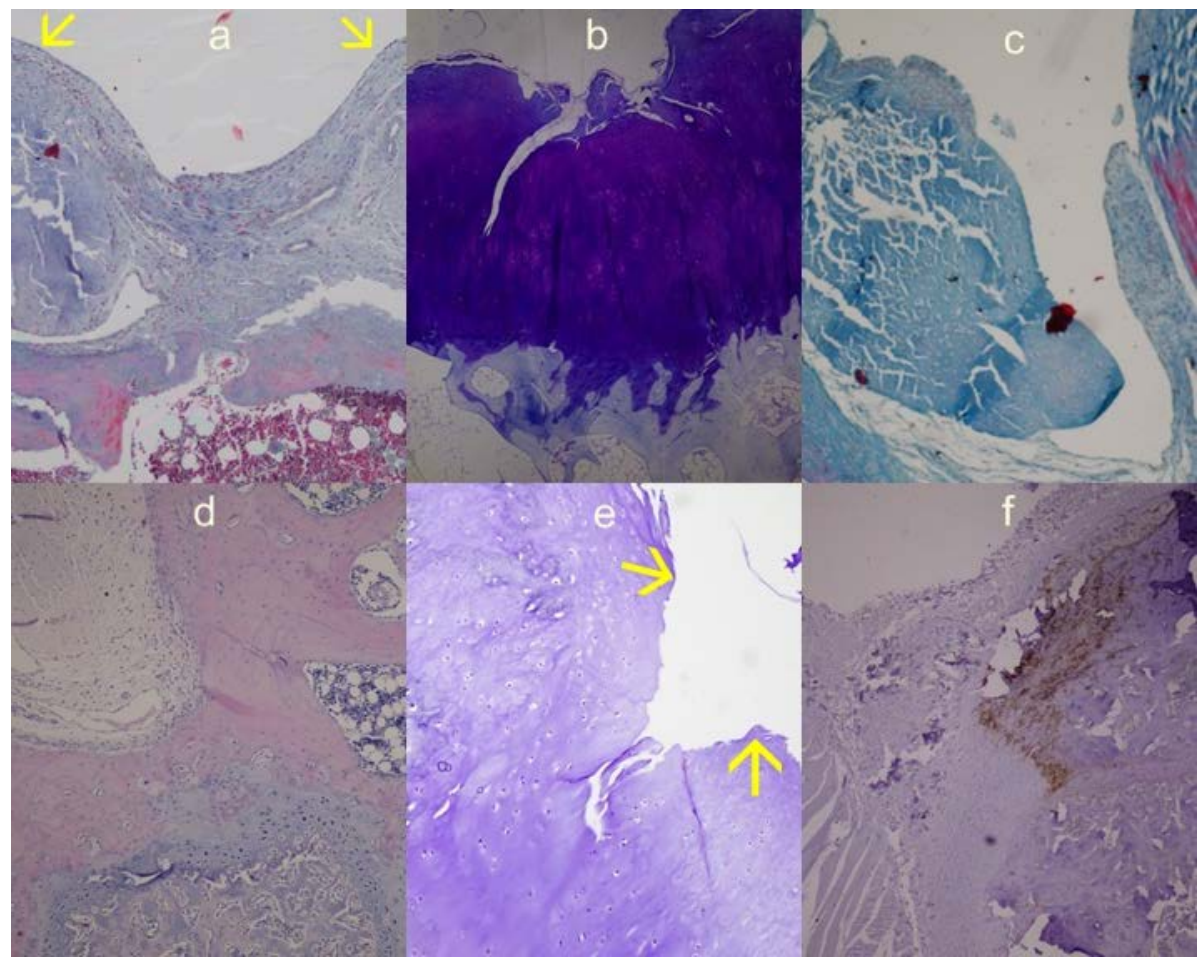

Figure 5. (a-f) Histological assessment of Group A. (a) Defects healed to 50-75\% with fibrocartilage; Masson trichrome $(\times 10)$ and (b) Toluidine blue $(\times 20)$. (c) Partial healing of the PRF-treated defect; Masson trichrome $(\times 40)$ and (d) Hyaline cartilage-filled defect; hematoxylin $\&$ eosin $(\times 20)$. (e) Unhealed defect; Toluidine blue $(\times 40)$ and (f) a partly healed defect; collagen II $(\times 20)$.

\section{DISCUSSION}

The main goal of studies that investigate cartilage healing is to obtain faster production of and better quality hyaline cartilage. With autogenous cartilage grafts, there is a risk of donor area morbidity. Moreover, homogeneous and xenografts have the potential of causing immunological and infectious problems. The focus of this study was to obtain easily produced and reliable biomaterials that accelerate 
cartilage healing. The goal was to achieve biomechanical functioning similar to that of normal joint cartilage, with a minimally invasive procedure [12]. Platelet-rich preparations are appealing, as they are autogenous and contain many growth factors [13, 14]. Indeed, the most important components of tissue repair are growth factors, which are released during injury from platelet granules [15]. After a microfracture, subchondral bone haemorrhage occurs, and the fibrin network and platelets aid in recovery; however, these effects are limited. Thus, use of PRF is preferred, as it provides abundant platelets. PRF is an inexpensive and reliable biomaterial used in various disciplines $[16,17]$. In the current study, we observed hyaline cartilage in the joint treated with PRP at the end of 8 weeks. The smoothness of the repair surface and proximity to the normal thickness of the cartilage have resulted in high scores for the PRF group. Kazemi et al. [18] treated the cartilage defect in dogs with the PRF. Although histological and macroscopic scores were higher but results not statistically significant. Hamanishi et al. [19] observed hyaline cartilage after 4 weeks with atelocollagen membrane after drilling. Same study showed that 4 weeks after creation of the defects showed positive immunoreactivity of transforming growth factor beta (TGFb) in the deep layer of fibrous reparative tissue above the area of ingrowth of hyaline cartilage [19].

In our study, osteochondral defects were created in the right and left knees of rats, and only the right knees were treated with PRF. The healing periods of experimental models using different animal groups vary, and a follow-up period of 1-12 months is recommended for adequate macroscopic and histological evaluations [18, 20, 21]. Performing studies in rats is easy, and it provides opportunities for histological examination. However, macroscopic examination may not be possible in rodent studies. Recent studies have achieved satisfactory histological results after study durations of 4 and 12 weeks in rat knee cartilage models $[19,22]$. In our study, we demonstrated that injured rat cartilage and subchondral bone healed within 2 months. Significant improvements were observed in the PRF group at 8 weeks, but no significant differences between the PRF and control groups were found during at 4 weeks, according to O'Driscoll scores. A defect of $1.5 \mathrm{~mm}$ in diameter is considered large in the rat knee joint;
Jackson et al. [23] showed that $3 \mathrm{~mm}$ diameter defects did not heal spontaneously in sheep knees. Vasara et al. [20] obtained spontaneous chondral healing without any augmentation material in injured immature porcine knee joints. In our study, no spontaneous filling of the defects occurred in the control group. However, fibrocartilage was observed in all sections studied, even in the PRF-treated group. Due to the smooth surface of the cartilage and complete filling of the defect, the PRF group received high scores. The average defect-filling rate in the control group ranged from 50 to $75 \%$. In a previous study, Kuo et al. [24] showed that PRF improved the healing of cartilage defects. It is desirable to have a defect filled with cartilage with smooth healing.

\section{Limitations}

Our study is not without limitations. First, a larger number of experimental animals should be used to perform International Cartilage Repair Society scoring. A defect greater than $1.5 \mathrm{~mm}$ could be created. Moreover, a longer follow-up period would have enabled us to better demonstrate the stages and the evolution of cartilage healing. The results of human PRF applications may not be similar to those of rats, and histologic findings may not be correlated with functional outcomes.

\section{CONCLUSION}

PRF is a reliable and effective biomaterial for use in the recovery of cartilage defects, as it contains growth factors, cellular material, and a fibrin skeleton.

\section{Conflict of interest}

The authors disclosed no conflict of interest during the preparation or publication of this manuscript.

\section{Funding Statement}

This audit was supported by a grant from the Scottish Government Whole System Patient Flow programme.

\section{REFERENCES}

[1] Sophia Fox AJ, Bedi A, Rodeo SA. The basic science of 
articular cartilage: structure, composition, and function. Sports Health 2009;1:461-8.

[2] Buma P, Pieper JS, Van Tienen T, Van Susante JL, Van der Kraan PM, Veerkamp JH, et al. Cross-linked type I and type II collagenous matrices for the repair of full-thickness articular cartilage defects: a study in rabbits. Biomaterials 2003;24:325563.

[3] Dohan Ehrenfest DM, Rasmusson L, Albrektsson T. Classification of platelet concentrates: from pure platelet-rich plasma (P-PRP) to leucocyte- and platelet-rich fibrin (L-PRF). Trends Biotechnol 2009;27:158-67.

[4] Choukroun J, Adda F, Schoeffler C, Vervelle A. [An opportunity in perio-implantology: the PRF]. Implantodontie 2001;42:55-62. [Article in French]

[5] Choukroun J, Diss A, Simonpieri A, Girard MO, Schoeffler C, Dohan SL, et al. Platelet-rich fibrin (PRF): A secondgeneration platelet concentrate. Part IV: Clinical effects on tissue healing. Oral Surg Oral Med Oral Pathol Oral Radiol Endod 2006;101:e56-60.

[6] Choukroun J, Diss A, Simonpieri A, Girard MO, Schoeffler C, Dohan SL, et al. Platelet-rich fibrin (PRF): a secondgeneration platelet concentrate. Part V: Histologic evaluations of PRF effects on bone allograft maturation in sinus lift. Oral Surg Oral Med Oral Pathol Oral Radiol Endod 2006;101: 299-303.

[7] Dohan DM, Choukroun J, Diss A, Dohan SL, Dohan AJ, Mouhyi J, et al. Platelet-rich fibrin (PRF): a second-generation platelet concentrate. Part II: Platelet-related biologic features. Oral Surg Oral Med Oral Pathol Oral Radiol Endod 2006;101:e45-50.

[8] Dohan DM, Choukroun J, Diss A, Dohan SL, Dohan AJ, Mouhyi J, et al. Platelet-rich fibrin (PRF): a second-generation platelet concentrate. Part I: Technological concepts and evolution. Oral Surg Oral Med Oral Pathol Oral Radiol Endod 2006;101:e37-44.

[9] Masuki H, Okudera T, Watanebe T, Suzuki M, Nishiyama K, Okudera H, et al. Growth factor and pro-inflammatory cytokine contents in platelet-rich plasma (PRP), plasma rich in growth factors (PRGF), advanced platelet-rich fibrin (A-PRF), and concentrated growth factors (CGF). Int J Implant Dent 2016;2:19. [10] He L, Lin Y, Hu X, Zhang Y, Wu H. A comparative study of platelet-rich fibrin (PRF) and platelet-rich plasma (PRP) on the effect of proliferation and differentiation of rat osteoblasts in vitro. Oral Surg Oral Med Oral Pathol Oral Radiol Endod 2009;108:707-13.

[11] O'Driscoll SW, Keeley FW, Salter RB. The chondrogenic potential of free autogenous periosteal grafts for biological resurfacing of major full-thickness defects in joint surfaces under the influence of continuous passive motion. An experimental investigation in the rabbit. J Bone Joint Surg Am 1986;68:101735 .

[12] Stone KR, Walgenbach AW, Freyer A, Turek TJ, Speer DP.
Articular cartilage paste grafting to full-thickness articular cartilage knee joint lesions: a 2- to 12- year follow-up. Arthroscopy 2006;22:291-9.

[13] Su CY, Kuo YP, Tseng YH, Su CH, Burnouf T. In vitro release of growth factors from platelet-rich fibrin (PRF): a proposal to optimize the clinical applications of PRF. Oral Surg Oral Med Oral Pathol Oral Radiol Endod 2009;108:56-61.

[14] Kang YH, Jeon SH, Park JY, Chung JH, Choung YH, Choung HW, et al. Platelet-rich fibrin is a bioscaffold and reservoir of growth factors for tissue regeneration. Tissue Eng Part A 2011;17:349-59.

[15] Sampson S, Gerhardt M, Mandelbaum B. Platelet rich plasma injection grafts for musculoskeletal injuries: a review. Cur Rev Musculoskelet Med 2008;1:165-74.

[16] Zumstein MA, Berger S, Schober M, Boileau P, Nyffeler RW, Horn M, et al. Leukocyte- and platelet-rich fibrin (L-PRF) for long-term delivery of growth factor in rotator cuff repair: review, preliminary results and future directions. Curr Pharm Biotechnol 2012;13:1196-206.

[17] Roy S, Driggs J, Elgharably H, Biswas S, Findley M, Khanna S, et al. Platelet-rich fibrin matrix improves wound angiogenesis via inducing endothelial cell proliferation. Wound Repair Regen 2011;19:753-66.

[18] Kazemi D, Fakhrjou A, Dizaji VM, Alishahi MK. Effect of autologous platelet rich fibrin on the healing of experimental articular cartilage defects of the knee in an animal model. Biomed Res Int 2014;2014:486436.

[19] Hamanishi M1, Nakasa T, Kamei N, Kazusa H, Kamei G, Ochi M. Treatment of cartilage defects by subchondral drilling combined with covering with Atelocollagen membrane induces osteogenesis in a rat model. J Orthop Sci 2013;18:627-35.

[20] Vasara AI, Hyttinen MM, Pulliainen O, Lammi MJ, Jurvelin JS, Peterson L, et al. Immature porcine knee cartilage lesions show good healing with or without autologous chondrocyte transplantation. Osteoarthritis Cartilage 2006;14:1066-74.

[21] Hurtig MB, Buschmann MD, Fortier LA, Hoemann CD, Hunziker EB, Jurvelin JS, et al. Preclinical studies for cartilage repair: recommendations from the International Cartilage Repair Society. Cartilage 2011;2:137-52.

[22] Dahlin RL, Kinard LA, Lam J, Needham CJ, Lu S, Kasper FK, et al. Articular chondrocytes and mesenchymal stem cells seeded on biodegradable scaffolds for the repair of cartilage in a rat osteochondral defect model. Biomaterials 2014;35:7460-9.

[23] Jackson DW, Lalor PA, Aberman HM, Simon TM. Spontaneous repair of full thickness defects of articular cartilage in a goat model. A preliminary study. J Bone Joint Surg Am 2001;83:53-64.

[24] Kuo TF, Lin MF, Lin YH, Lin YC, Su RJ, Lin HW, et al. Implantation of platelet rich fibrin and cartilage granules facilitates cartilage repair in the injured rabbit knee: preliminary report. Clinics (Sao Paulo) 2011;66:1835-8. 